\title{
Sechs Kategorien genügen bei der Handekzem-Diagnostik
}

\author{
Für die genauere Diagnostik von Handekzem-Patienten ist kein kompliziertes Regelwerk nötig: \\ Sechs Untergruppen reichen, um rund $90 \%$ der Patienten präzise einzuordnen, so das Ergebnis \\ einer Veröffentlichung im Journal of the European Academy of Dermatology and Venereology.
}

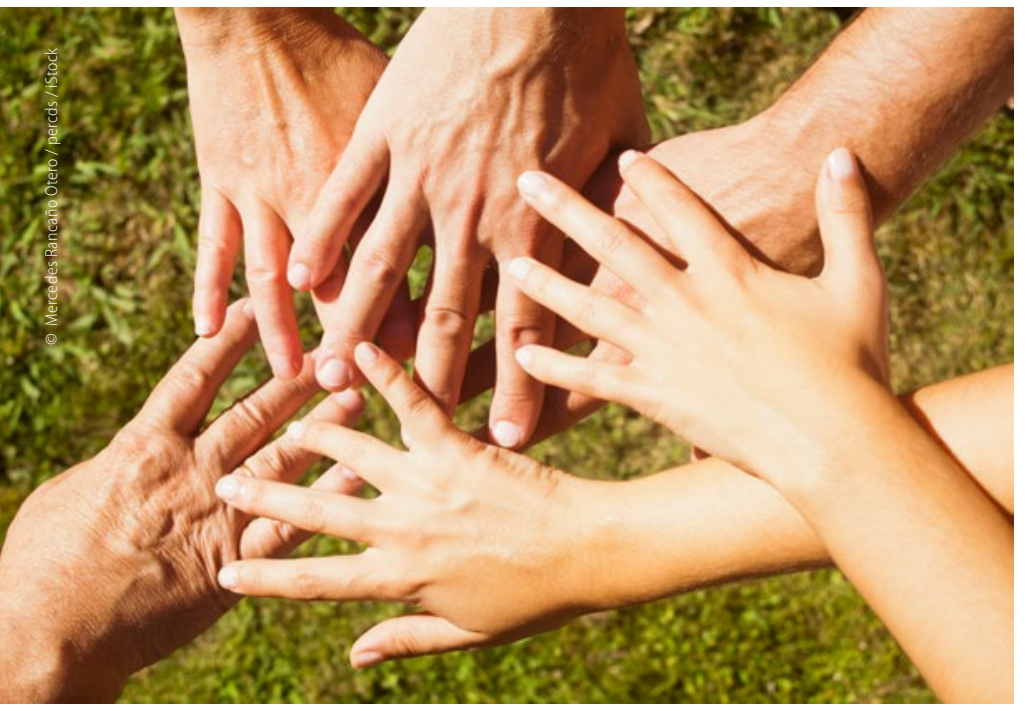

9 von 10 Patienten mit einem Handekzem können mithilfe eines sechsstufigen Klassifikationsschemas diagnostisch eingeteilt werden.

Für Patienten mit Handekzem ist eine korrekte Diagnostik mitunter sehr wichtig - nicht nur um die Prognose abzuschätzen, die richtige Therapie zu wählen und auslösende Faktoren zu vermeiden, sondern auch bei Rechtstreitigkeiten aufgrund von berufsbedingten Ekzemen. Allerdings ist eine genaue Diagnose nicht immer leicht, da sich das klinische Bild im Laufe der Erkrankung oft stark ändert und nicht unbedingt mit den auslösenden Faktoren korreliert.

Um die Diagnostik zu vereinfachen, haben Dermatologen um Tove Agner von der Universität in Kopenhagen vor Kurzem ein neues Klassifikationsschema vorgeschlagen. Dies haben sie nun in einer Praxisstudie getestet. Teilgenommen haben 427 Patienten mit einem Handekzem, die an neun dermatologische Zentren in mehreren europäischen Ländern überwiesen wurden. Die folgende Einteilung haben die Dermatologen für die Diagnostik verwendet:

1. Allergische Kontaktdermatitis: Voraussetzung hierfür ist ein positiver Patchtest auf ein Allergen oder ein kreuzreagierendes Allergen sowie ein dokumentierter oder angenommener Handkontakt mit dem jeweiligen Allergen.

2. Irritative Kontaktdermatitis: Der Kontakt mit größeren Mengen eines Ekzem-auslösenden Reizstoffs muss belegt werden. In Frage kommen vor allem Detergenzien bei häufigem Händewa- schen (über 20-mal täglich), auch das Tragen von Handschuhen über mehrere Stunden hinweg ist ein Indiz. Eine allergische Kontaktdermatitis sollte ausgeschlossen werden.

3. Atopisches Handekzem: Dieses ist zu vermuten, wenn Patienten in der Vergangenheit bereits atopische Ekzeme hatten und Reizstoffe als Auslöser ausgeschlossen werden können.

4. Protein-Kontaktdermatitis/Kontakturtikaria: Hierbei lassen sich biologische Stoffe wie Latex oder Nahrungsmittel als Auslöser über einen Pricktest nachweisen. Auch eine spezifische IgE-Reaktion auf verdächtiges Material bestätigt die Diagnose. Die Patienten haben oft auch atopische Ekzeme.

5. Vesikulär-endogenes Handekzem: Kann ein atopisches Ekzem ausgeschlossen werden und lassen sich keine Trigger finden, ist eine Orientierung am klinischen Bild möglich. Bei der vaskulär-endogenen Form stehen bläschenförmige Eruptionen im Vordergrund.

6. Hyperkeratotisch-endogenes Handekzem: Bläschen und Pusteln werden bei dieser Form nicht beobachtet, dafür eine Pulpitis oder eine Hyperkeratose auf der Handinnenseite.

Wie sich herausstellte, konnten die Ärzte $89 \%$ der Patienten direkt einer der genannten Kategorien zuordnen. Ein Drittel hatte eine irritative und ein Viertel eine allergische Kontaktdermatitis, $12 \%$ hatten ein atopisches Ekzem und $3 \%$ eine ProteinKontaktdermatitis/Kontakturtikaria. Bei knapp $10 \%$ griff die Diagnose hyperkeratotisch-endogen, bei $6 \%$ vesikulär-endogen.

Nur $4 \%$ ließen sich überhaupt nicht klassifizieren, bei einem Teil davon bestand der Verdacht auf eine Psoriasis. Bei rund 7\% erschien eine einzige Kategorie nicht angebracht, diese Patienten erhielten zwei oder mehr Hauptdiagnosen. Am häufigsten war hierbei die Kombination irritative plus allergische Kontaktdermatitis. Bei 36\% aller Patienten kamen die Ärzte mit der Hauptdiagnose aus, bei $38 \%$ stellten sie eine Haupt- und eine Nebendiagnose, bei $26 \%$ zwei oder mehr Nebendiagnosen. Dies spiegele die oft multifaktorielle Ätiologie bei Handekzemen wider, schreiben die Studienautoren. Etwa die Hälfte der Patienten kam während ihrer Arbeit mit dem Ekzemauslöser in Kontakt.

Insgesamt sehen die Dermatologen anhand ihrer Studie ausreichend Hinweise, dass das vorgeschlagene Klassifikationssystem auch für den Praxiseinsatz taugt.

Thomas Müller

Agner T et al. J Eur Acad Dermatol Venereol 2015, Sep 15; doi:10.1111/jdv.13308 